\title{
Utilidad de la linfadenectomía en el cáncer de vejiga. Revisión de la literatura
}

\author{
Monzó JI, Herranz Amo F, Cabello Benavente R, Hernández Fernández C. \\ Servicio de Urología. Hospital General Universitario Gregorio Marañón. Madrid.
}

Actas Urol Esp. 2007;31(1):1-6

\section{RESUMEN}

\section{UTILIDAD DE LA LINFADENECTOMÍA EN EL CÁNCER DE VEJIGA. REVISIÓN DE LA LITERATURA}

Objetivos: Revisión de la utilidad de la linfadenectomía en el cáncer de vejiga.

Material y Métodos: Revisión en la base de datos Medline/PubMed de los artículos publicados en los últimos 10 años, según las siguientes palabras claves: "bladder cancer, lymphadenectomy, lymph node metastasis". Seleccionándose 33 artículos que cumplian los criterios de selección.

Conclusiones: No existe ningún trabajo prospectivo y aleatorizado que compare las diferentes técnicas de linfadenectomía pélvica. La afectación ganglionar global en el cáncer de vejiga después de cistectomía y linfadenectomía varía desde un $18 \%$ hasta un $28 \%$.

La realización de una linfadenectomía al menos del tipo estándar mejoraría la estadificación tumoral y es probable que también la supervivencia en determinados pacientes. La extracción de al menos 10-14 ganglios en la linfadenectomía parece recomendable como estándar de calidad. La densidad de ganglios positivos parece ser mejor predictor de la supervivencia libre de enfermedad que el sistema clásico TNM.

Se recomienda la linfadenectomía por separado a la linfadenectomía en bloque porque proporciona mayor número de ganglios.

Palabras clave: Cáncer de vejiga. Linfadenectomía. Metástasis ganglionar.

\section{ABSTRACT \\ THE USEFULNESS OF PELVIC LYMPHADENECTOMY IN BLADDER CANCER}

Objetive: To assess the usefulness of pelvic lymphadenectomy in bladder cancer.

Methods and Results: With the followings key words: "bladder cancer, lymphadenectomy, lymph node metastasis" we search in Medline/PubMed database for papers published during the last ten years. Thirty three papers fulfilling the search criteria were selected.

Conclusions: It does not exist any randomized prospective study comparing different pelvic lymphadenectomy techniques. Nodal metastasis in bladder cancer after radical cystectomy and pelvic lymphadenectomy ranged between $18 \%$ and $28 \%$. Standard lymphadenectomy could improve tumor staging and probably survival in selected patients. It is advisable to remove, as a quality parameter, at least 10 to 14 nodes in a pelvic lymphadenectomy. Lymph node density seems to predict survival better than TNM staging system. It is advisable to perform separate lymph node dissection rather than en-bloc.

Keywords: Bladder cancer. Lymphadenectomy. Lymph node metastasis.

$\mathrm{D}$ ebido a la ausencia de estudios prospectivos aleatorizados que comparen el resultado oncológico entre la cistectomía radical y la cistectomía simple en el cáncer de vejiga, son muchos los interrogantes sobre la utilidad de la linfade- nectomía pélvica en dicha patología. No obstante, una vez decidido realizarla, encontramos diferentes opiniones sobre la amplitud de la disección linfática y la valoración de la información que brinda. En manos expertas no se encuentran 
mayores complicaciones cuando se aumenta la amplitud de la disección linfática ${ }^{1-3}$ ni tampoco aumenta considerablemente el tiempo quirúrgico.

La linfadenectomía proporciona información esencial para la estadificación tumoral y por lo tanto, sobre el pronóstico del paciente. Es probable que en pacientes con baja carga tumoral ganglionar la linfadenectomía pueda tener algún efecto en la supervivencia, pero no existe ningún estudio aleatorizado que confirme este hecho.

\section{OBJETIVOS}

Revisión de la utilidad de la linfadenectomía en el cáncer de vejiga.

\section{MATERIAL Y MÉTODOS}

Hemos realizado una búsqueda bibliográfica en Medline/PubMed según los siguientes criterios:

- Dirección de búsqueda: http://www.ncbi. nlm.nih.gov/entrez/query.fcgi?DB=pubmed.

- Fecha de revisión: mayo de 2006.

- Periodo de revisión: últimos 10 años.

- Palabras claves: bladder cancer, lymphadenectomy, lymph node metastasis.

- Idiomas: español e ingles.

Esquema de trabajo:

- Lectura detallada de los resúmenes de todos los artículos obtenidos.

- Selección de aquellos trabajos que se ajustaban a los diferentes temas que se fijaron para la revisión, a saber:

1. Localización de las metástasis ganglionares.

2. Técnicas de linfadenectomía.

3. Comparación entre las diferentes técnicas de linfadenectomía

4. Número de ganglios a resecar en la linfadenectomía y su aplicación.

5. Concepto de densidad de ganglios positivos.

6. Diferencias entre linfadenectomía en bloque y por separado.

\section{RESULTADOS}

Mediante la combinación de las diferentes palabras claves se obtuvieron 162 artículos de los cuales se seleccionaron 33. Se descartaron 61 trabajos cuyos contenidos no desarrollaban ninguno de los criterios de selección, 6 trabajos sobre radiología, 15 de investigación básica, 2 sobre mortalidad perioperatoria, 8 de tratamiento con quimioterapia, 12 sobre tumores distintos al de células transicionales, 24 de cirugía laparoscópica y 1 publicado en una revista egipcia sin poder acceder a dicha publicación. No encontramos ningún ensayo clínico aleatorizado. Doce revisiones aparecen hasta la fecha indicada ${ }^{4-15}$.

Incidencia y localización de las metástasis ganglionares en el cáncer de vejiga.

El drenaje linfático vesical se realiza a través de tres grupos distintos de conductos linfáticos denominados anterior, lateral y posterior. El grupo anterior termina en uno o dos ganglios linfáticos situados sobre la vena iliaca externa, el grupo lateral lo hace en los ganglios iliacos externos e hipogástricos y el posterior en los ganglios situados por debajo de la bifurcación aórtica drenando fundamentalmente el territorio trigonal y la cara posterior vesical ${ }^{16}$.

La afectación ganglionar global en el cáncer de vejiga varía desde un $18 \%$ hasta un $28 \%$ (Tabla 1). En un estudio descriptivo sobre la localización de la afectación ganglionar en 176 pacientes a los que se les realizo linfadenectomía pélvica, la afectación global de ganglios perivesicales fue del $22,7 \%$, en ganglios iliacos comunes del $18 \%$, en ganglios presacros del $5,1 \%$ y por encima de la bifurcación aórtica del 4\%. Esta distribución metastásica variaba al estratificar según el estadio tumoral ${ }^{17}$. En el mismo trabajo se observó que sólo el 3,6\% de los tumores pT1 tuvieron ganglios positivos, todos localizados en la pelvis.

Tabla 1

Incidencia de la invasión ganglionar en los pacientes con cáncer de vejiga tratados mediante cistectomía radical

\begin{tabular}{lccc}
\hline & Año & Casos & N+(\%) \\
\hline Ghoneim $^{32}$ & 1997 & 1.026 & $188(19,5 \%)$ \\
Vieweg $^{30}$ & 1999 & 682 & $193(28,1 \%)$ \\
Herr and Donat $^{33}$ & 2001 & 763 & $196(25,0 \%)$ \\
Mills $^{28}$ & 2001 & 452 & $83(18)$ \\
Stein $^{29}$ & 2003 & 1.054 & $246(24 \%)$ \\
Vazina $^{17}$ & 2004 & 176 & $43(24,4 \%)$ \\
Abdel Latif & & & \\
\hline
\end{tabular}


Sólo un 3\% de los pT2 tuvieron afectación ganglionar fuera de la pelvis y del tejido perivesical. Un $26 \%$ de los tumores pT3 y pT4 frecuentemente presentaron enfermedad fuera de los límites de la linfadenectomía estándar (LE). Leissner et al. ${ }^{2}$ en 290 pacientes a los que se les realizó linfadenectomía pélvica definieron tres niveles de linfadenectomía. El nivel I se extendía hasta la bifurcación iliaca, el nivel II hasta la bifurcación aórtica y por ultimo el nivel III hasta el origen de la mesentérica inferior. El 21\% presentaron afectación en el nivel I, $18,6 \%$ en el nivel II y $6,6 \%$ en el último nivel. Además un $6.9 \%$ tuvieron metástasis únicamente en el nivel I, el 6,9\% sólo en el nivel II y ninguno tuvo afectación aislada en el nivel III. Esto demuestra que hasta casi un $7 \%$ de las metástasis pueden aparecer por fuera de los limites de la linfadenectomía limitada (LL) y la linfadenectomía estándar (LE) que definiremos más adelante. De los pacientes con metástasis en el nivel I, el 57\% presentaron afectación en el nivel II y el 31\% en el nivel III. Los ganglios en el nivel III se encontraron sólo si las metástasis estaban presentes en 9 o más ganglios en el nivel I o II.

En cuanto a los factores de riesgo para la afectación ganglionar, el estadio tumoral, el grado de diferenciación y la invasión linfovascular son factores pronósticos independientes ${ }^{18}$.

\section{Extensión de la linfadenectomía}

Según la extensión craneal de la linfadenectomía se han descrito tres técnicas de linfadenectomía:

- Linfadenectomía Limitada (LL): incluye los grupos ganglionares obturador e hipogástrico y los ganglios perivesicales.

- Linfadenectomía Estándar (LE): incluye los grupos de la LL más el grupo ganglionar de la arteria y vena ilíaca externa, nervio genitofemoral y ligamento inguinal.

- Linfadenectomía Ampliada (LA): similar a los grupos de la estándar más el grupo ganglionar de la arteria iliaca común y los ganglios presacros $^{19}$.

¿Existe evidencia científica a favor de una u otra técnica de linfadenectomía?

Como comentamos anteriormente no existe ningún estudio prospectivo aleatorizado compa- rando una técnica con otra. Tampoco existe ningún estudio controlado que demuestre el valor curativo de la linfadenectomía por lo que las guías clínicas de la EAU recomiendan la $\mathrm{LL}^{20}$.

Poulsen et al. ${ }^{1}$ en un análisis retrospectivo compararon 126 pacientes a los que se les realizó LA con 68 tratados con LL, obtuvieron una mejoría en la supervivencia libre de recurrencia a 5 años a favor de la LA $(85 \%$ vs $64 \%$ p $<0,02)$ para los estadios $\leq$ pT3a con una supervivencia global del $75 \%$. En los tumores $\geq$ pT3b no se encontraron diferencias estadísticamente significativas. En el mismo trabajo la LA reducía el riesgo de recurrencia pélvica a 5 años $(2 \%$ vs $7 \%$ p=0,17) y la probabilidad de metástasis a distancia (10\% vs $21 \% \mathrm{p}<0,15$ ) en los tumores limitados a la pared vesical. Cuando se estratifica por afectación ganglionar demuestran una ventaja similar en cuanto a supervivencia para los pacientes NO. La supervivencia a 5 años en este grupo fue del 90\% para estadio $\leq$ pT3a cuando se les realizó L.A. comparado con un $71 \%$ en la L.L. $(\mathrm{p}<0,02)$.

En un trabajo aleatorizado con 270 pacientes a los que se les realizó cistectomía radical se observó que la supervivencia fue del 33\%, 46\% y 60\% cuando se realizó LL, LE y LA respectivamente. En el análisis multivariante, la amplitud de la disección linfática, el número de ganglios resecados y el número de casos realizados por el cirujano resultaron factores significativos que influyeron en la supervivencia ${ }^{21}$.

Datos a favor de disecciones más amplias también se pueden observar en trabajos como el de Leissner et al. ${ }^{2}$ quienes observaron que si la linfadenectomía se limitaba al espacio obturador se sobreexplorarian un $6,8 \%$ de los pacientes, pero el $74,1 \%$ de todos los ganglios positivos quedarían sin identificar. Además no encuentran ninguna correlación entre el número de ganglios positivos y el número de ganglios resecados por paciente.

Knap et al. encontraron una mejoría en la supervivencia cuando se extraen más de 3 ganglios (p: 0,02), por lo que concluyen que la cistectomía radical con linfadenectomía de tipo limitada resulta insuficiente y debería realizarse una linfadenectomía pélvica más extensa ${ }^{22}$.

Para Bochner et al..$^{23}$ ambas técnicas identifican un porcentaje similar de ganglios positivos (21\%). 
Según Konety et al. ${ }^{24}$, analizando la base de datos del Surveillance, Epidemiology and End Results (SEER), existe una correlación positiva entre el numero de ganglios examinados y la supervivencia $(r: 0,2 \mathrm{p}<0,0001)$ para pacientes en estadio IV, pero no así entre el numero de ganglios positivos y la supervivencia (r:-0,08 $\mathrm{p}=0,1466$ ). Según los resultados obtenidos en el mismo trabajo la linfadenectomía pélvica tiene un efecto significativo en la supervivencia libre de enfermedad en pacientes con neoplasia vesical primaria.

Herr $^{25}$ refiere que cuanto mayor es el número de ganglios extraídos mayor es el número de pacientes detectados con afectación ganglionar. Esta asociación tiene una Correlación de Spearman muy débil de 0,17 (p=001). Abdel Latif et al. ${ }^{18}$ encuentran una correlación de 0,1 para la misma asociación $(r=0,4 \mathrm{p}<0,001)$.

\section{¿Qué impacto tiene el número de ganglios} resecados y cuántos ganglios debe el cirujano obtener para considerar una linfadenectomía como suficiente?

Leissner et al. $^{26}$ evaluaron 447 pacientes a los que se les realizó cistectomía radical más linfadenectomía pélvica pero sólo 302 tuvieron seguimiento. Encontraron diferencias en la supervivencia cáncer específica $(\mathrm{p}<0,013)$ y en el intervalo libre de enfermedad $(p<0,016)$ entre los pacientes con $\geq 16$ ganglios resecados comparados con $\leq 15$. Esta diferencia fue pequeña durante los primeros 20 meses del seguimiento, pero a los 5 años el $65 \%$ de los pacientes con $\geq 16$ ganglios estaban libres de enfermedad, mientras que sólo el $51 \%$ de los pacientes con $\leq 15$ ganglios estaban en las mismas condiciones. El intervalo libre de enfermedad para pacientes con tumores pT1G3, pT2a y pT2b con NO fue significativamente mejor cuando se resecaron $\geq 16$ ganglios comparados con $\leq 15$ ( $85 \%$ vs $65 \% \mathrm{p}<0,01)$. Esto se explicaría, probablemente, por la remoción de micrometástasis no detectadas por el patólogo. Herr et al. ${ }^{27}$ observaron que el número de ganglios tiene un impacto significativo en el control local y en la supervivencia en estadio pNO y pN+. Estos autores recomiendan extraer al menos 9 ganglios.

Mills et al. ${ }^{28}$ analizaron prospectivamente 452 cistectomías radicales con linfadenectomía pélvica con una media de 20 ganglios resecados.
Encontraron diferencias en cuanto a la supervivencia cuando existían menos de 5 ganglios afectados al compararlo con más de 5 (27 meses frente a 15 meses $p=0,0027$ ). La perforación capsular aumentaba más del doble el riesgo de muerte $(\mathrm{HR}=2,6)$ y el tamaño de la metástasis parecía condicionar la supervivencia.

Para Stein ${ }^{29}$ el número total de ganglios resecados proveía información pronóstica pero no alcanzaba un nivel estadísticamente significativo. Esto se observó comparando la supervivencia libre de recurrencia a 5 y 10 años entre $\geq 15$ ó $<15$ ganglios resecados (36\% frente a $25 \% \mathrm{p}=0,21)$.

Konety et al. ${ }^{24}$ observaron que la disminución más alta en el riesgo de muerte fue para el grupo de 10 a 14 ganglios resecados (HR riesgo de muerte $0,38 \mathrm{IC}=95 \%(0,24-0,57))$ comparado con 1-3, 1-6, 7-9 ó 15-19 ganglios resecados, recomendando junto con Herr ${ }^{19}$ la resección de al menos 10-14 ganglios como parámetro de calidad para la linfadenectomía.

Leissner ${ }^{2}$ propone un promedio de 43 ganglios resecados como medida de calidad de la linfadenectomía.

¿Qué es y qué utilidad tiene el concepto de densidad de ganglios positivos?

La relación entre el número de ganglios positivos y el número total de ganglios resecados define al concepto de densidad de ganglios positivos.

El sistema TNM clasifica la afectación ganglionar en función del número y el tamaño de los ganglios afectados, pero no describe la carga tumoral en relación a la extensión o calidad de la disección ganglionar. Vieweg et al. ${ }^{30}$ encuentran correlación entre la supervivencia libre de enfermedad y el estadio N. Para N1, N2 y N3 la supervivencia libre de enfermedad a los 3 años fue del $50,6 \%, 34,5 \%$ y $0 \%$ respectivamente. Herr observó que la densidad de ganglios positivos pronostica mejor la supervivencia y el control local que el sistema clásico TNM y no encuentra correlación entre los diferentes estadios $\mathrm{N}$ y la supervivencia. El mismo autor fija un punto de corte del $20 \%$ a partir del cual encuentra diferencias estadisticamente significativas en la supervivencia. Tanto para la supervivencia específica como para la recurrencia local, la densidad de ganglios positivos es una variable pronóstica independiente ${ }^{25}$. 
Konety et al. ${ }^{24}$ encontraron que a medida que aumentaba esta relación disminuía la supervivencia pero no fijan un punto de corte.

Stein et al. ${ }^{29}$ fijando también un punto de corte del $20 \%$ observaron que la supervivencia libre de enfermedad y supervivencia global a $5 \mathrm{y}$ 10 años para una densidad $<20 \%$ es del $44 \%$ y $43 \%$ respectivamente, comparado con un $17 \%$ para ambos plazos de tiempo $(\mathrm{p}<0,01)$ con una densidad $>20 \%$.

\section{¿Existen diferencias en cuanto al número de} ganglios extraídos entre la linfadenectomía por separado y la linfadenectomía en bloque?

Un único trabajo fue desarrollado para comparar ambas modalidades de linfadenectomía. Se obtuvieron una media de 2,4 ganglios con la linfadenectomía en bloque y una media de 8,5 con la linfadenectomía por separado $(\mathrm{p}=0,003)^{31}$. Leissner ${ }^{2}$ también obtuvo mejores resultados con la linfadenectomía por separado.

\section{CONCLUSIONES}

- No existe ningún trabajo prospectivo y aleatorizado que compare las diferentes técnicas de linfadenectomía pélvica.

- La afectación ganglionar global en el cáncer de vejiga después de cistectomía y linfadenectomía varía desde un $18 \%$ hasta un $28 \%$.

- La realización de una linfadenectomía al menos del tipo estándar mejoraría la estadificación tumoral y es probable que también la supervivencia en determinados pacientes.

- La extracción de al menos 10-14 ganglios en la linfadenectomía parece recomendable como estándar de calidad.

- La densidad de ganglios positivos parece ser mejor predictor de la supervivencia libre de enfermedad que el sistema clásico TNM.

- Se recomienda la linfadenectomía por separado de cada región ganglionar a la linfadenectomía en bloque porque proporciona mayor número de ganglios.

\section{REFERENCIAS}

1. Poulsen AL, Horn T, Steven K. Radical cystectomy: extending the limits of pelvic lymph node dissection improves survival for patients with bladder cancer confined to the bladder wall. J Urol. 1998;160(6 Pt 1):2015-2019.
2. Leissner J, Ghoneim MA, Abol-Eneim H, Thüroff JW, Fisch FM, Schulze $\mathrm{H}$ et al. Extended radical lymphadenectomy in patients with urothelial baldder cancer: results of a prospective multicenter study. J Urol. 2004;171(1):139-144.

3. Brossner C, Pycha A, Toth A, Mian C, Kuber W. Does extended lymphadenectomy increase the morbidity of radical cystectomy? BJU Int. 2004;93(1):64-66.

4. Ghoneim MA, Abol-Eneim H. Lymphadenectomy with Cystectomy: Is It Necessary and What Is Its Extent? Eur Urol. 2004;46(4):457-461.

5. Stein JP, Skinner DG. The role of lymphadenectomy in high-grade invasive bladder cancer. Urol Clin North Am. 2005; 32(2): 187-197.

6. Huang WC, Bochner BH. Current status of establishing standards for lymphadenectomy in the treatment of bladder cancer. Curr Opin Urol. 2005;15(5):315-319.

7. Busby JE, Evans CP. Old friends, new ways: revisiting extended lymphadenectomy and neoadjuvant chemotherapy to improve outcomes. Curr Opin Urol. 2004;14(5): 251-257.

8. Sanderson KM, Stein JP, Skinner DG. The evolving role of pelvic lymphadenectomy in the treatment of bladder cancer. Urol Oncol. 2004;22(3):205-211.

9. Tsukamoto T, Kitamura H, Takahashi A, Masumori N. Treatment of invasive bladder cancer: Lessons from the past and Perspective for the Future. Jpn J Clin Oncol. 2004;34(6):295-306.

10. Simms MS, Mann G, Kockelbergh RC, Mellon JK. The management of lymph node metastasis from bladder cancer. Eur J Cancer Surg. 2005;31(4):348-356.

11. Liedberg F, Mansson W. Lymph node metastasis in bladder cancer. Eur Urol. 2006;49(1):13-21.

12. Ather MH, Fatima S, Sinanoglu O. Extent of lymphadenectomy in radical cystectomy for bladder cancer. WJ Surg Oncol. 2005; 15(3):43.

13. Stein JP, Quek ML, Skinner DG. Lymphadenectomy for invasive bladder cancer: I. historical perspective and contemporary rationale. BJU 2006;97(2):227-231.

14. Stein JP, Quek ML, Skinner DG. Lymphadenectomy for invasive bladder cancer: II. Technical aspects and prognostic factors. BJU 2006;97(2):232-237.

15. Quek ML, Sanderson KM, Daneshmand S, Stein JP. The importance of an extended lymphadenectomy in the management of high-grade invasivebladder cancer. Expert Rev. Anticancer Ther. 2004;4(6):1007-1016.

16. Testut L, Latarjet A. Tratado de Anatomia Humana. Novena Edición. Salvat Editores. 1954.

17. Vazina A, Dugi D, Shariat SF, Evans J, Link R, Lerner S. Stage specific lymph node metastasis mapping in radical cystectomy specimens. J Urol. 2004;171(5):1830-1834.

18. Abdel-Latif M, Abol-Eneim H, El-Baz M, Ghoneim MA. Nodal involvement in bladder cancer cases treated with radical cystectomy: incidente and prognosis. J Urol. 2004; 172(1):85-89.

19. Herr H, Lee C, Chang S, Lerner S. Standardization of radical cystectomy and pelvic lymph node dissection for bladder cancer: a collaborative group report. J Urol. 2004; 171(5): 1823-1828.

20. European Urology Association. Guidelines on bladder cancer 2004.

21. Herr HW. Surgical factors in baldder cancer: more (nodes) + more (pathology) = less (mortality). BJU 2003;92(3):187188. 
22. Knap MM, Lundbeck F, Overgaard J. The role of pelvic node dissection as predictive and prognostic factor in bladdercancer. European J Cancer 2003;39(5):604-613.

23. Bochner BH, Cho D, Herr HW, Donat M, Kattan MW, Dalbagni G. Prospectively packaged lymph node dissections with radical cystectomy: evaluation of node count variability and node mapping. J Urol. 2004;172(4 Pt 1):1286-1290.

24. Konety BR, Joslyn SA, O`Donell MA. Extent of pelvic lymphadenectomy and its impact on outcome in patients diagnosed with bladder cancer: analysis of data from the surveillance, epidemiology and end results program data base. J Urol. 2003;169(3):946-950.

25. Herr HW. Superiority of ratio based lymph node staging for bladder cancer. J Urol. 2003;169(3):943-945.

26. Leissner J, Hohenfellner R, Thüroff JW, Wolf HK. Lymphadenectomy in patients with transitional cell carcinoma of the urinary bladder; significance for staging and prognosis. BJU 2000;85(7):817-823.

27. Herr HW, Bochner BH, Dalbagni G, Donat SM, Reuter VE, Bajorin DF. Impact of the number of lymph nodes retrived on outcome in patients with muscle invasive bladder cancer. J Urol. 2002;167(3):1295-1298.

28. Mills RD, Turner WH, Fleischmann A, Marhwalder G, Thalmann GN, Studer UE. Pelvic lymph node metastases from bladder cancer: outcome in 83 patients after radical cystectomy and pelvic lymphadenectomy. J Urol. 2001; 166(1):19-23.
29. Stein JP, Cai J, Groshen S, Skinner DG. Risk factors for patients with lymph node metastases following radical cystectomy with en bloc pelvic lymphadenectomy: the concept of lymph node density. J Urol. 2003;170(1):3541.

30. Vieweg J, Gschwend $\mathrm{J}$ E, Herr H W. The impact of primary stage on survival in patients with lymph node positive bladder cancer. J Urol. 1999;161(1):72-76.

31. Bochner BH, Herr HW, Reuter VE. Impact of separate versus en bloc pelvic lymph node dissection on the number of lymph nodes retrieved in cystectomy specimens. J Urol. 2001;166(6):2295-2296

32. Ghoneim MA, El-Mekresh MM, El-Baz MA, El-Attar IA, Ashamallah A. Radical cystectomy for carcinoma of the bladder: critical evaluation of the results in 1026 cases. J Urol. 1997;158(2):393-399.

33. Herr HW, Donat SM. Outcome of patients with grossly node positive bladder cancer after pelvic lymph node dissection and radical cystectomy. J Urol. 2001;165(1):62-64.

Correspondencia autor: Dr. JI Monzó. Servicio de Urología Hospital Gregorio Marañón. Dr. Esquerdo, 46. 28007 Madrid. Tel.: 915868000

E-mail autor: juanimonzo@hotmail.com

Información artículo: Revisión - Cáncer vesical

(Trabajo aceptado septiembre 2006) 